\title{
Paliperidone ER: a review of the clinical trial data
}

\author{
Philip G Janicak' \\ Elizabeth A Winans ${ }^{2,3}$ \\ 'Department of Psychiatry, Rush \\ University Medical Center, Chicago, \\ IL, USA; 'Previously Employed by \\ Scientific Affairs, Ortho-McNeil \\ Janssen, LLC, Chicago, IL, USA; \\ ${ }^{3}$ Department of Pharmacy Practice, \\ University of Missouri Kansas City, \\ MO, USA
}

\begin{abstract}
Paliperidone extended-release tablet (paliperidone ER; INVEGA ${ }^{\mathrm{TM}}$ ) is an oral antipsychotic for the treatment of schizophrenia. The recommended dose range is $3-12 \mathrm{mg}$ per day. Paliperidone ER utilizes the OROS $^{\circledR}$ delivery system, which allows for once-daily dosing. Its pharmacokinetic profile results in a more stable serum concentration. Paliperidone is 9-hydroxyrisperidone, the chief active metabolite of risperidone. It undergoes limited hepatic metabolism, thereby minimizing the risks of hepatic drug-drug and drug-disease interactions. Three 6-week trials in patients with acute schizophrenia reported that paliperidone ER was effective, well tolerated, and produced clinically meaningful improvements in personal and social functioning compared with placebo. Post-hoc analysis of these trials in various populations, including recently diagnosed, elderly and more severely ill patients, those with sleep disturbances and those with predominant negative symptoms demonstrated improvement as well. Paliperidone ER was also significantly better than placebo in the prevention of symptom recurrence in a 6-month maintenance study. The most common clinically relevant adverse events associated with paliperidone ER were extrapyramidal symptoms, tachycardia and somnolence. The incidence of Parkinsonism, akathisia and use of anticholinergic medications increased in a dose-related manner. Further, modest QTc interval prolongation was observed but did not produce clinical symptoms. Similar to risperidone, paliperidone ER is associated with increases in serum prolactin levels. Overall, paliperidone ER was effective, well tolerated and provides a new treatment option for patients with schizophrenia.
\end{abstract}

Keywords: paliperidone, extended-release, antipsychotic, schizophrenia

\section{Introduction}

Schizophrenia is a chronic disorder that affects about $1 \%$ of the world's population. While presently available antipsychotics can ameliorate an acute exacerbation of positive symptoms (eg, hallucinations and delusions), their benefit for deficit, cognitive and mood symptoms are not as pronounced. Furthermore, the safety and tolerability of each agent may differ substantially, often determining the clinician's initial choice of drug and patients' willingness to adhere to treatment (Janicak et al 2006). Paliperidone extended-release tablet (paliperidone ER) (INVEGA ${ }^{\mathrm{TM}}$, Johnson \& Johnson Pharmaceuticals, Titusville, NJ, USA), the 9-OH metabolite of risperidone, has recently received approval by the United States (US) Food and Drug Administration (FDA) and by the European Medicines Evaluation Agency (EMEA). This agent combines the efficacy of risperidone with an innovative, osmotically-controlled oral delivery system called OROS ${ }^{\mathrm{TM}}$, which releases the drug at a controlled rate specific to the properties of 9-OH risperidone (Conley et al 2006). This allows for once-daily dosing and can minimize the 24-hour peak-to-trough variation at steady state concentration $\left(\mathrm{C}_{\mathrm{SS}}\right)$. Furthermore, its limited hepatic metabolism may reduce drug-drug or drug-disease interactions and its novel delivery system allows for a drug-specific controlled release that produces a more sustained, even exposure over time. While 9-OH risperidone has been identified as an important component of risperidone's effects, it has not been studied previously as an antipsychotic for schizophrenia. 
The purpose of this article is to review the pharmacodynamic, pharmacokinetic, efficacy and safety/tolerability profile of paliperidone ER, the major active metabolite of risperidone.

\section{Methods}

The background data to provide this review were derived from a search of PUBMED, EMBASE, and several international congresses (between January 2004 and May 2007) using the key word "paliperidone". It should be noted that many of the studies discussed are presently available only in abstract form and have not been peer reviewed. The three acute pivotal studies and recurrence study, however, have all been accepted or published in peer-reviewed journals.

\section{Results}

\section{OROS formulation}

Paliperidone ER is formulated within an osmotically controlled release oral delivery system called OROS. The OROS formulation delivers paliperidone at a controlled rate over a 24-hour period. The system consists of an osmotically active trilayer core surrounded by a semi-permeable membrane. The trilayer core contains two drug layers and a push compartment (Figure 1). After administration, water passes through the semi-permeable membrane, thereby controlling the rate of passage into the tablet membrane core, which in turn, controls drug delivery (Conley et al 2006). The OROS technology results in reduced fluctuations between drug peak and trough serum concentrations (eg, 38\% paliperidone ER versus $125 \%$ risperidone immediate-release [IR]) (Rossenu et al 2007). To preserve the integrity of the OROS delivery system, the tablet should be swallowed whole and not chewed, split or crushed (INVEGA package insert 2007). Since the shell of the tablet is non-absorbable, prescribers should inform patients that the undissolved residue may be observed in their stool.

\section{Pharmacodynamics/pharmacokinetics of paliperidone ER}

Pharmacodynamics

Paliperidone is a benzisoxazole derivative that demonstrates high affinity for central dopamine ${ }_{2}$ and serotonin $_{2 \mathrm{~A}}$ receptors. In addition, it has affinity for both alpha-adrenergic ${ }_{1 \text { and }}$ ${ }_{2}$ and histaminic ${ }_{1}$ receptors. Paliperidone does not possess affinity for muscarinic-cholinergic and beta-adrenergic
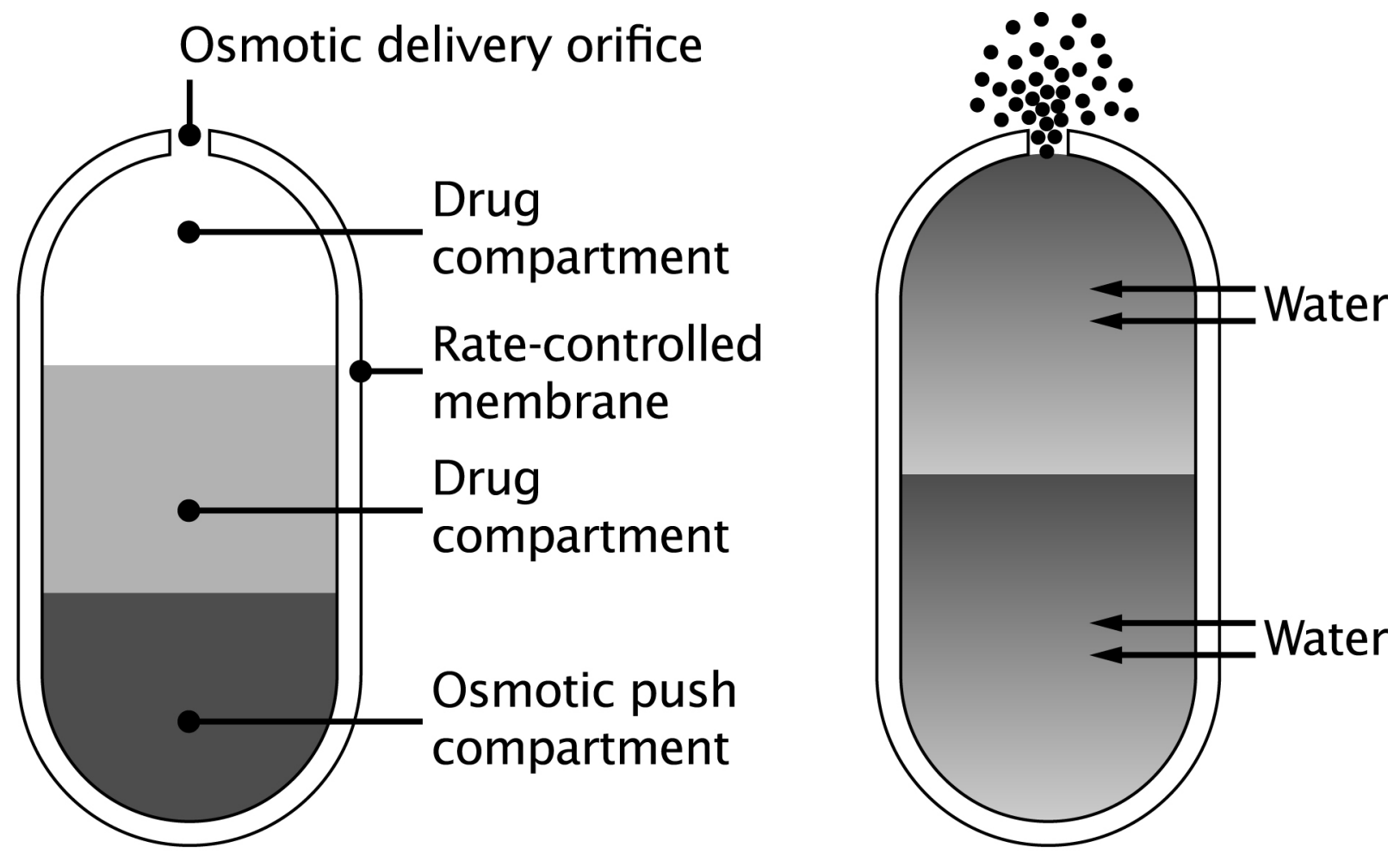

\section{Before operation}

During operation

Figure I OROS delivery system. The osmotic push-pull tablet: cross-section of bilayer tablet before and after ingestion. Reprinted with permission from Conley R, Gupta SK, Sathyan G. 2006. Clinical spectrum of the osmotic-controlled release oral delivery system (OROS ${ }^{\circledR}$ ), an advanced oral delivery form. Curr Med Res Opin, 22:I879-92. Copyright (c) 2006 Informa Healthcare. 
receptors. In vitro, the enantiomers ( + and - ) of paliperidone demonstrate similar pharmacologic activity.

\section{Pharmacokinetics}

The absolute oral bioavailability of paliperidone ER is $28 \%$ (INVEGA package insert 2007). Administration of this agent after a high-fat or high-calorie meal increased the maximum serum concentration and area under the curve values by $60 \%$ and $54 \%$, respectively. While paliperidone ER can be taken without regard to meals, the presence of food may increase its exposure (INVEGA package insert 2007). Patients in the clinical efficacy trials, however, were dosed without regard to meal timing. The terminal halflife of paliperidone ER is about 23 hours with steady state concentration attained in 4-5 days (Rossenu et al 2006; INVEGA package insert 2007).

Paliperidone ER is widely and rapidly distributed after oral administration. It is $74 \%$ protein bound, primarily to albumin and $\alpha 1$-acid glycoprotein. Paliperidone ER undergoes very limited hepatic metabolism, with approximately $60 \%$ of the unchanged drug eliminated renally and $11 \%$ eliminated unchanged in the feces (Vermeir et al 2005). Paliperidone ER does not appear to possess enzyme-inducing or enzyme-inhibiting properties as shown by the lack of CYP 450 inhibition in in vitro studies in human liver microsome studies (INVEGA package insert 2007). Thus, it is less prone to hepatic drug-drug or drug-disease interactions. While paliperidone ER undergoes both active and passive renal elimination, its co-administration with trimethoprim, a potent organic cation inhibitor, did not significantly alter its clearance rates (Thyssen et al 2006a). Overall, the paliperidone ER pharmacokinetic profile demonstrates dose proportionality within the recommended clinical range of 3-12 $\mathrm{mg} /$ day (Rossenu et al 2006).

\section{Hepatic impairment}

There may be a higher incidence of liver problems (eg, alcohol-related; hepatitis C) in patients with schizophrenia compared with the general population (Carney et al 2006). In this context, the pharmacokinetic parameters of paliperidone ER were also assessed in patients with moderate hepatic impairment compared with healthy controls (Thyssen et al 2006b). There were minimal differences in the terminal elimination half-life between these two groups (26.5 hours for hepatically impaired patients versus 23.6 hours for healthy subjects). In addition, there was a small reduction in the total exposure to paliperidone ER in the hepatically impaired patients. After correcting for reduced $\alpha 1$-acid glycoprotein binding secondary to hepatic impairment, however, the total exposure to paliperidone ER was similar between groups. The unbound paliperidone ER levels were slightly lower in the hepatically impaired group as compared with healthy controls. As the unbound drug is most relevant to safety and efficacy, no dosage adjustment is recommended in these patients.

\section{Renal impairment}

The dose of paliperidone ER should be lowered in patients with moderate-to-severe renal impairment, since the elimination of paliperidone decreases with decreasing estimated creatinine clearance (INVEGA package insert 2007; Thyssen et al 2007). The exact dose of paliperidone ER should be individualized according to the patient's renal function status. For patients with mild renal impairment (with a creatinine clearance of $\geq 50$ to $<80 \mathrm{~mL} / \mathrm{min}$ ), the maximum recommended dose is $6 \mathrm{mg}$ paliperidone ER once daily. For patients with moderate-to-severe renal impairment (with a creatinine clearance of 10 to $<50 \mathrm{~mL} / \mathrm{min}$ ), the maximum recommended dose is $3 \mathrm{mg}$ paliperidone ER once daily.

\section{Efficacy in acute trials}

The acute efficacy of paliperidone ER has been examined in three studies (Davidson et al 2007; Kane et al 2007; Marder et al 2007). These studies involved a 6-week (at least 14 days as an inpatient), double-blind, randomized, fixed-dose, placebo- and active-controlled (olanzapine $10 \mathrm{mg}$ per day), parallel-group design. The primary outcome measure in all studies was the mean change in total Positive and Negative Syndrome Scale (PANSS) score. Secondary outcome measures included the Marder PANSS factor scores (Marder et al 1997), response rates, Clinical Global Impressions-Severity (CGI-S) scores (Guy 1976) and Personal and Social Performance (PSP) scale scores (Morosini et al 2000; Patrick et al 2006). This last scale allows clinicians to rate personal and social functioning on a 100-point scale in 10-point increments (eg, 1-10 representing lack of autonomy in basic functioning; 91-100 representing excellent functioning). The scale considers four domains of behavior: socially useful activities, relationships, self-care, and disturbing and aggressive behaviors. Improvement by one category (ie, 10 points) reflects a clinically meaningful change.

Marder et al conducted the first study at 74 US centers (Marder et al 2007). It included patients with chronic schizophrenia who were experiencing an acute exacerbation (ie, PANSS score range $=70-120 ;$ mean \pm standard deviation $[S D]$ baseline score $=94 \pm 12$ ) of their disorder. Four hundred and forty-four subjects were randomized and 192 (43\%) completed 
Table I Patient demographics and baseline characteristics in the intent-to-treat population

\begin{tabular}{|c|c|c|c|c|c|}
\hline & $\begin{array}{l}\text { Placebo } \\
(n=105)\end{array}$ & $\begin{array}{l}\text { Paliperidone ER } \\
6 \mathrm{mg} \\
(\mathrm{n}=\mathrm{III})\end{array}$ & $\begin{array}{l}\text { Paliperidone ER } \\
\text { I } 2 \mathrm{mg} \\
(\mathrm{n}=\mathrm{II} \mathrm{I})\end{array}$ & $\begin{array}{l}\text { Olanzapine } \\
10 \mathrm{mg} \\
(\mathrm{n}=105)\end{array}$ & $\begin{array}{l}\text { Total } \\
(n=432)\end{array}$ \\
\hline Age (years) ${ }^{\mathrm{a}}$ & $42.3(10.7)$ & $42.1(10.2)$ & $41.4(10.7)$ & $40.5(11.0)$ & $41.6(10.7)$ \\
\hline \multicolumn{6}{|l|}{ Sex (\%) } \\
\hline Male & 78 & 68 & 69 & 80 & 74 \\
\hline Female & 22 & 32 & 31 & 20 & 26 \\
\hline \multicolumn{6}{|l|}{ Race (\%) } \\
\hline White & 48 & 41 & 41 & 42 & 43 \\
\hline Black & 50 & 58 & 59 & 53 & 55 \\
\hline Asian & 0 & 0 & 0 & 4 & I \\
\hline Other & 2 & I & I & I & I \\
\hline Weight (kg) ${ }^{\mathrm{a}}$ & $89.7(20.3)$ & $87.4(19.4)$ & $87.0(21.6)$ & $89.7(23.2)$ & $88.4(2||)$. \\
\hline PANSS total score ${ }^{a}$ & $93.6(11.7)$ & $92.3(12.0)$ & $94.1(11.4)$ & $94.9(12.4)$ & $93.7(11.9)$ \\
\hline
\end{tabular}

aMean (standard deviation).

Abbreviations: ER, extended release; PANSS, Positive and Negative Syndrome Scale.

Adapted with permission from Marder SR, Kramer M, Ford L et al 2007. Efficacy and safety of paliperidone extended-release tablets: results of a 6-week, randomized, placebo-controlled study. Biol Psychiatry, June 28; [Epub ahead of print] doi:10.1016/j.biopsych.2007.01.017. Copyright (C) 2007 Elsevier.

the study. The treatment arms included paliperidone ER ( 6 or $12 \mathrm{mg}$ fixed doses), placebo, and olanzapine $(10 \mathrm{mg})$. Since the olanzapine arm was an active control group to confirm assay sensitivity, it was not included in the statistical models for efficacy analyses. The demographic and baseline characteristics of the 432 intent-to-treat (ITT) patients (ie, randomized patients who received $\geq 1$ dose of double-blind study drug and had $\geq 1$ post-baseline efficacy measure) were similar across all groups (see Table 1). Rescue medications (primarily lorazepam) were used by approximately $75 \%$ of subjects in each group for a mean duration of approximately 8 days. The primary outcome measure was change in the PANSS total score from baseline to end point. Both doses of paliperidone ER separated to a statistically significant degree in comparison with placebo ( $6 \mathrm{mg}$ dose, $\mathrm{p}=0.006 ; 12 \mathrm{mg}$ dose, $\mathrm{p}<0.001$ ). There was also a significant difference from placebo at every post-baseline rating from Day 4 onward in the $6 \mathrm{mg}$ dose group $(\mathrm{p}<0.05)$, and from Day 15 onward in the $12 \mathrm{mg}$ dose group $(\mathrm{p}<0.05)$. Clinical response rates (defined by $\geq 30 \%$ improvement from the baseline PANSS total score) were also significantly higher in the paliperidone groups versus the placebo group ( $6 \mathrm{mg}=$ $50 \%[\mathrm{p}<0.03] ; 12 \mathrm{mg}=51 \%[\mathrm{p}=0.012]$; placebo $=34 \%)$. By comparison, the response rate in the olanzapine $(10 \mathrm{mg})$ group was approximately $46 \%$.

Substantially fewer subjects on paliperidone ER were classified as "marked/severe/extremely severe" on the CGI-S scale at end point compared with baseline $(6 \mathrm{mg}=57.6 \%$ to $26.1 \% ; 12 \mathrm{mg}=64.0 \%$ to $20.7 \%$; placebo $=60.0 \%$ to $44.7 \%$ ). By comparison, the olanzapine (10 mg) group went from $70.5 \%$ to $29.6 \%$. While PSP scale scores improved in both paliperidone ER dose groups, only the $6 \mathrm{mg}$ dose separated from placebo to a statistically significant degree $(\mathrm{p}=0.007)$.

The second trial by Kane et al was similar to the Marder study but had some important design differences. These included the involvement of sites in both the US and India and three fixed doses of paliperidone ER (6, 9, and $12 \mathrm{mg}$ ). Six hundred and thirty patients entered and 415 (66\%) completed the study (Kane et al 2007). Demographic and baseline characteristics, as well as use and duration of rescue medications (mainly lorazepam), were similar across the four treatment arms. All three doses of paliperidone ER separated to a statistically significant degree from placebo based on the mean change in the PANSS total $(p<0.001)$ and all five Marder factor scores from baseline to end point $(\mathrm{p}<0.001)$. Mean PANSS total change scores with paliperidone ER were significantly greater than placebo for the $12 \mathrm{mg}$ dose from Day 4 onward $(\mathrm{p}<0.01)$, and for the $6 \mathrm{mg}$ and $9 \mathrm{mg}$ doses from Day 8 onward $(\mathrm{p}<0.05)$ (Figure 2). Response rates were almost double in the paliperidone ER groups $(6 \mathrm{mg}=56 \% ; 9 \mathrm{mg}=51 \% ; 12 \mathrm{mg}=61 \%)$ versus placebo $(30 \% ; \mathrm{p}<0.001)$. By comparison, the response rate in the olanzapine (10 mg) group was $52 \%$.

Rates of discontinuation due to lack of efficacy were also lower in the paliperidone ER versus placebo group $(6 \mathrm{mg}=16 \% ; 9 \mathrm{mg}=16 \% ; 12 \mathrm{mg}=10 \%$; placebo $=40 \%)$. A substantially lower percentage of subjects were classified as "marked/severe/extremely severe" on the CGI-S score from baseline to end point (paliperidone: $6 \mathrm{mg}=63 \%$ to $21 \%$; $9 \mathrm{mg}=57 \%$ to $23 \%$; $12 \mathrm{mg}=64 \%$ to $16 \%)$ compared with placebo $(60 \%$ to $51 \%)$. By comparison, the olanzapine $(10 \mathrm{mg})$ group went from 

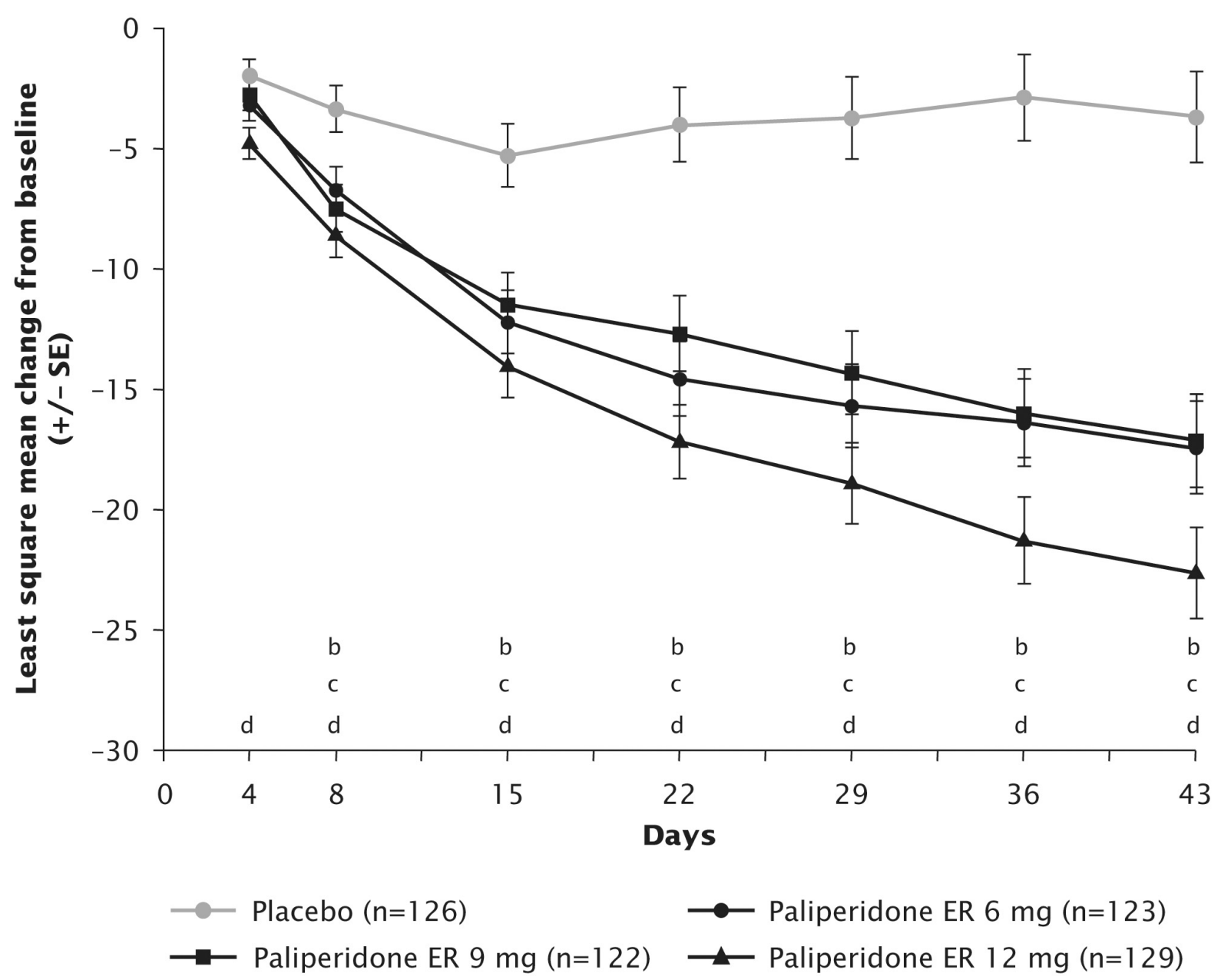

Figure 2 Change in positive and negative symptom scale total score over time in the intent-to-treat population (Kane et al. 2007). Improvement in mean PANSS total score for paliperidone ER versus placebo was statistically significant at every post-baseline time point from Day 4 (first observation point) for paliperidone ER I 2 mg ( $<0.0$ I versus change in placebo group), and from Day 8 for the paliperidone ER 6 and $9 \mathrm{mg}$ groups ( $<0.05$ versus change in placebo group). This was maintained for the remainder

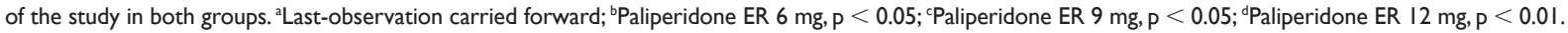
Abbreviations: ER, extended-release; SE, standard error.

Reprinted with permission from Kane J, Canas F, Kramer M et al 2007. Treatment of schizophrenia with paliperidone extended-release tablets:A 6-week placebo-controlled trial. Schizophrenia Res, 90:147-6I.Copyright @ 2007 Elsevier.

$64 \%$ at baseline to $24 \%$ at end point. Finally, PSP scale scores improved significantly from baseline to end point for all three doses of paliperidone ER compared with placebo $(\mathrm{p}<0.001)$ (Figure 3).

The third study by Davidson et al was also similar to the Marder and Kane studies with the following important differences. This was a multi-center, international trial that included three fixed doses of paliperidone ER ( $3 \mathrm{mg}, 9 \mathrm{mg}$, and $15 \mathrm{mg}$ ). Six hundred and eighteen patients were randomized and 365 (59\%) completed the study (Davidson et al 2007). As in the Marder et al and Kane et al trials, demographic and baseline characteristics, as well as the use and duration of rescue medications, were similar across all groups.

All paliperidone ER doses produced significantly greater improvements in the PANSS total and Marder factor scores at end point versus placebo $(\mathrm{p}<0.01)$. All three doses demonstrated statistically significant improvement from placebo by Day 4 onward. More than twice as many patients in all three paliperidone ER groups achieved a clinical response at end point versus placebo ( $3 \mathrm{mg}=40 \%$; $9 \mathrm{mg}=46 \% ; 15 \mathrm{mg}=53 \%$; placebo $=18 \%$; $\mathrm{p} \leq 0.005)$. By comparison, the proportion of responders in the olanzapine (10 mg) active control group at end point was $52 \%$. Rates of discontinuation due to lack of efficacy were lower in the paliperidone ER groups compared with placebo and decreased in a dose-related manner (ie, $3 \mathrm{mg}=24 \%$; $9 \mathrm{mg}=18 \%$; $15 \mathrm{mg}=12 \%$; placebo $=44 \%$ ). By comparison, the olanzapine (10 mg) active control group had a $13 \%$ discontinuation rate due to inefficacy.

A significant improvement in the mean PSP scale scores $( \pm \mathrm{SD})$ from baseline to end point was also seen for all three doses of paliperidone ER versus placebo ( $3 \mathrm{mg}=8.3 \pm 17.1$; 


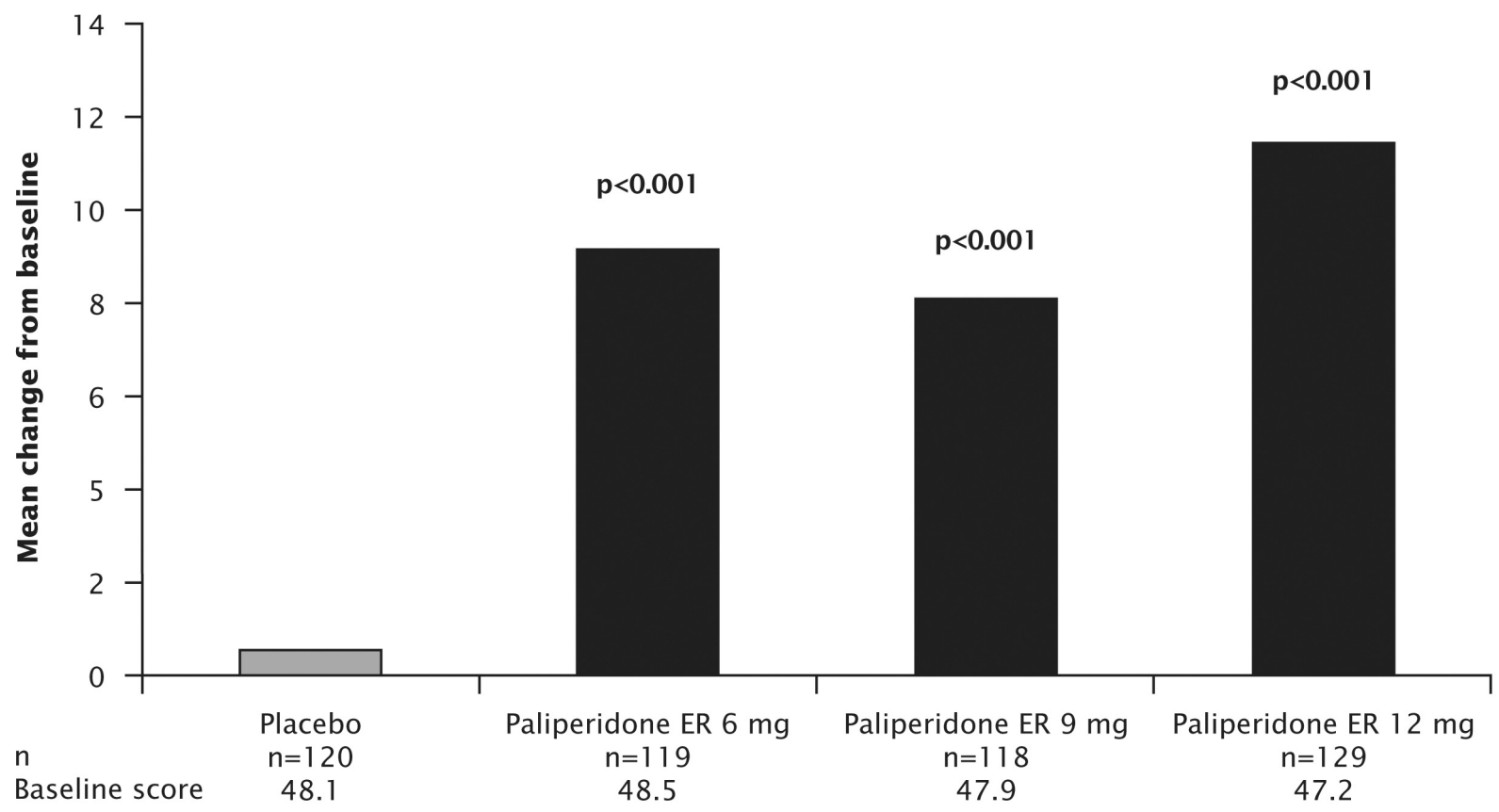

Figure 3 Improvements in social and personal functioning, measured using the Personal and Social Performance scale in the intent-to-treat population ${ }^{\text {a }}$ (Kane et al 2007). Personal and Social Performance scale scores improved significantly from baseline to end point for all the paliperidone ER groups versus placebo ( $P<0.00 \mathrm{I}$ for all doses). a Last-observation carried forward.

Abbreviations: ER, extended-release.

$9 \mathrm{mg}=7.6 \pm 14.2 ; 15 \mathrm{mg}=12.2 \pm 15.7 ;$ placebo $=-1.5 \pm 15.8$; $\mathrm{p}<0.001)$. By comparison, the olanzapine $(10 \mathrm{mg})$ active control group improved $7.8 \pm 15.0$ points on the PSP scale.

Finally, substantially fewer patients in the paliperidone ER groups were classified as "marked/severe/extremely severe" from baseline to end point on the CGI-S scale versus the placebo group ( $3 \mathrm{mg}=54 \%$ to $32 \% ; 9 \mathrm{mg}=52 \%$ to $23 \% ; 15 \mathrm{mg}=57 \%$ to $17 \%$; placebo $=56 \%$ to $49 \%$ ). By comparison, the olanzapine (10 $\mathrm{mg}$ ) group went from $56 \%$ at baseline to $21 \%$ at end point.

An analysis of the pooled data from these three trials was conducted to clarify the main outcomes and detect any possible clinically meaningful trends (Meltzer et al 2006). The major finding indicated that, while all doses used in these trials $(3,6,9,12$, and $15 \mathrm{mg}$ ) were efficacious, the $3 \mathrm{mg}$ dose was least effective. Thus, paliperidone ER $6 \mathrm{mg}$ per day is the suggested starting dose for treatment of schizophrenia.

\section{Efficacy in specific populations and symptoms}

\section{Elderly patients with schizophrenia}

It is also important to assess the impact of antipsychotics in an older population given their high rates of polypharmacy, multiple co-morbid medical illnesses, age-related changes in metabolism, increased sensitivity to adverse events (AEs) and the potential for longer length of illness to compromise clinical efficacy. In this context, Tzimos et al conducted a 6-week, double-blind, randomized, flexible-dose, placebocontrolled trial in 114 patients with schizophrenia $\geq 65$ years (Tzimos et al 2007). The mean age of the subject population was $69.7 \pm 4.5$ years. The primary goal of this study was to assess the safety and tolerability of flexible doses (3-12 $\mathrm{mg}$ /day) of paliperidone ER compared with placebo. Initially, subjects were randomized to paliperidone ER $(6 \mathrm{mg} /$ day $)$ or placebo in a 2:1 ratio. After 1 week, the paliperidone ER dose was increased to $9 \mathrm{mg}$ /day (tolerability permitting) and, subsequently, flexibly adjusted between $3-12 \mathrm{mg}$ /day in $3 \mathrm{mg}$ dose increments or decrements. Relevant baseline clinical characteristics were similar between the two treatment arms. Eighty-four percent of the paliperidone ER group and $68 \%$ of the placebo group completed the study. Rescue medications (ie, benzodiazepines) were used in $26 \%$ of subjects in both groups. The mean modal dose of paliperidone ER was $8.3 \pm 3.0 \mathrm{mg} /$ day. Of note, treatment-emergent AEs (TEAEs) occurred in $67 \%$ of the active drug group and $71 \%$ of the placebo group. Serious AEs (SAEs) occurred in $3 \%$ of the active drug group and $8 \%$ of the placebo group. Two deaths (both in the placebo group) also occurred. In terms of cardiovascular, cerebrovascular, neuromotor and metabolic changes, only tachycardia was more prevalent in 
the paliperidone ER group (16\%) compared with the placebo group (0\%). This, however, did not lead to any clinically relevant events. Furthermore, improvements in the PANSS total and Marder factor scores, the CGI-S score, and percent of responders at end point all favored paliperidone ER over placebo. The authors concluded that paliperidone ER was safe, well tolerated and effective in reducing symptoms of schizophrenia in this older age group.

\section{Sleep symptoms}

Disrupted sleep is common in patients with schizophrenia and can cause subsequent daytime fatigue, precipitate exacerbations and prolong time to recovery. In this context, Luthringer et al (2007) conducted a randomized, double-blind, placebocontrolled, 3-week study in patients $(n=42)$ with schizophrenia-related insomnia to assess the effect of paliperidone ER (dosed Q AM) on sleep architecture. They also assessed the effect of this antipsychotic on patient-rated changes in sleep quality and daytime drowsiness based on the pooled data from the three pivotal trials. Overall, the data suggested that paliperidone ER had a positive effect on sleep architecture, continuity and patient-rated sleep quality without producing or worsening daytime sleepiness (Luthringer et al 2007).

\section{Patients recently diagnosed with schizophrenia}

There are two important considerations in managing patients who have been recently diagnosed with schizophrenia. The first is that early pharmacologic intervention may favorably alter the long-term prognosis by controlling symptoms, prolonging remission and preventing future episodes (or at least decreasing their frequency and severity). The second is that while patients early in the course of their illness may be more responsive to medication treatment, they may also be more sensitive to AEs. In this context, Kostic et al conducted a post hoc analysis of the data from the three, 6-week acute trials focusing on those patients diagnosed with schizophrenia within 5 years of study entry (Kostic et al 2006a, b). The data from patients diagnosed more than 5 years before study entry were also analyzed separately for reference to such key variables as baseline clinical characteristics and efficacy/tolerability end points (eg, PANSS, AEs). Four hundred and thirteen patients met the recently diagnosed criterion of $\leq 5$ years with the mean time ill being $2.8 \pm 1.4$ years for those on paliperidone ER (3-12 mg; $\mathrm{n}=284)$ and $2.8 \pm 1.3$ years for those on placebo $(n=100)$. Eight hundred and ninety-three patients were diagnosed $>5$ years prior to study entry. No differences in baseline demographics (with the exception of age and length of diagnosed illness) were evident between the two duration of illness groups. Completion rates and reasons for discontinuation also did not differ between the $\leq 5$ and $>5$ year duration of illness groups. Furthermore, both paliperidone ER duration of illness groups had about a $20 \%$ greater completion rate than the placebo group. Efficacy measures (PANSS total, Marder factor scores, CGI-S scores and PSP scale scores) were significantly improved from baseline in the $\leq 5$ year group compared with placebo. Changes in PANSS total score were similar to those observed in the $\geq 5$ year group (Kostic et al 2006a). These analyses suggest a comparable response in patients regardless of illness length. In addition, tolerability to paliperidone ER was similar between the two duration of illness groups, with the possible exceptions of greater vulnerability for movementdisorder-related effects (eg, akathisia and pseudoparkinsonism) and somnolence in those recently diagnosed. The authors note that potential limitations of these analyses include the post hoc method; assignment to the duration of illness group based on history; and the lack of comprehensive data on duration of undiagnosed and untreated psychosis.

\section{Severely ill patients with schizophrenia}

In a post hoc analysis of the pooled data from the three acute efficacy trials, Canuso and colleagues considered the impact of paliperidone ER (fixed doses of 3-12 mg per day) in those subjects who presented with "marked to severe" symptom levels (Canuso et al 2006b). The pre-defined criterion was a baseline PANSS total score $\geq 105$. Two hundred and seventeen patients qualified for the analysis with mean baseline PANSS scores of $111.4 \pm 5.7$ and $110.9 \pm 4.3$ in the active drug and placebo groups, respectively. At end point, the paliperidone ER group exhibited a significantly greater improvement $(\mathrm{p}<0.05)$ than the placebo group in the mean PANSS total score ( -26.7 versus -5.7 , respectively); CGI-S score ( -1.2 versus -0.1 , respectively); and all PANSS factor scores. The most frequent AEs in the paliperidone ER versus placebo group were headache (17\% versus $9 \%$, respectively), agitation ( $6 \%$ versus $13 \%$, respectively) and insomnia (14\% versus $14 \%$, respectively). Change scores on the Simpson Angus Scale (SAS) did not differ between groups. The authors concluded that paliperidone ER was effective and well tolerated in a more severely ill group of patients.

\section{Negative symptoms}

Deficit or negative symptoms can be a major impediment to recovery, ability to function and quality of life. These symptoms may be a primary manifestation of the illness or may occur as secondary manifestations in relation to other aspects 
of the disorder or its treatment (eg, affective symptoms and extrapyramidal symptoms [EPS]).

The impact of any treatment intervention may directly or indirectly affect negative symptoms. In this context, Canuso et al conducted a post hoc exploratory analysis of the pooled data from the three acute efficacy trials with paliperidone ER to assess its benefit for negative symptoms (Canuso et al 2006a). Two types of analyses were employed: regression analysis and path analysis. Regression analyses exploring the relationship between baseline/study characteristics and negative symptoms demonstrated that duration of drug exposure significantly predicted improvement in the PANSS negative symptom factor score $(\mathrm{p}<0.001)$. Furthermore, improvement in PSP scale scores was significantly associated with improvement in the PANSS negative symptom factor score $(\mathrm{p}<0.001)$. Path analysis models demonstrated that up to $33 \%$ of negative symptom improvement resulted from a direct effect of the drug, while the indirect effects were mediated through improvement in positive symptoms (51\%) and anxiety/depressive symptoms (18\%). Conversely, a $2.1 \%$ inverse effect on negative symptoms was accounted for by movement disorders as measured using the SAS (Figure 4). These analyses indicated a possible direct effect of paliperidone ER on negative symptoms.

In another post hoc analysis of this same data set, Dirks et al assessed the impact of paliperidone ER on patients with predominant negative symptoms (Dirks et al 2006). This was pre-defined as a baseline PANSS negative factor score $\geq 40 \%$ of the possible maximum plus a positive factor score $<40 \%$ of the possible maximum. Two hundred and ninety-nine patients met these criteria with an average of $49 \%$ of the maximum negative symptoms score and $33 \%$ of the maximum positive symptoms score. Those with and without predominant negative symptoms demonstrated comparable baseline demographic characteristics. Those with predominant negative symptoms demonstrated significant improvements following treatment with paliperidone ER compared with placebo on the primary and secondary outcome measures with no unexpected tolerability findings.

\section{Patients previously treated with risperidone or olanzapine}

Further, separate post hoc analyses of the pooled data from the three, 6-week acute trials assessed the efficacy of paliperidone ER compared with placebo in patients with acute schizophrenia who had been previously treated with risperidone $(n=198)$ or olanzapine $(n=153)$ immediately prior to study entry. The mean duration of risperidone treatment was $418.8 \pm 573$ days (paliperidone ER group) and $527.0 \pm 805.3$ (placebo group), with mean doses of $4.4 \pm 2.5 \mathrm{mg} /$ day and $4.1 \pm 2.6 \mathrm{mg} /$ day in the paliperidone ER and placebo groups, respectively. In the second analysis, the mean duration of olanzapine treatment was $490.1 \pm 677.7$ days for patients receiving paliperidone ER and $556.3 \pm 817.3$ days for those receiving placebo. Mean doses of olanzapine were $16.4 \pm 8.1 \mathrm{mg} /$ day and $17.0 \pm 6.2$ $\mathrm{mg}$ /day for patients receiving paliperidone ER and placebo, respectively. In both analyses, these patients experienced a significant improvement in mean PANSS total and factor

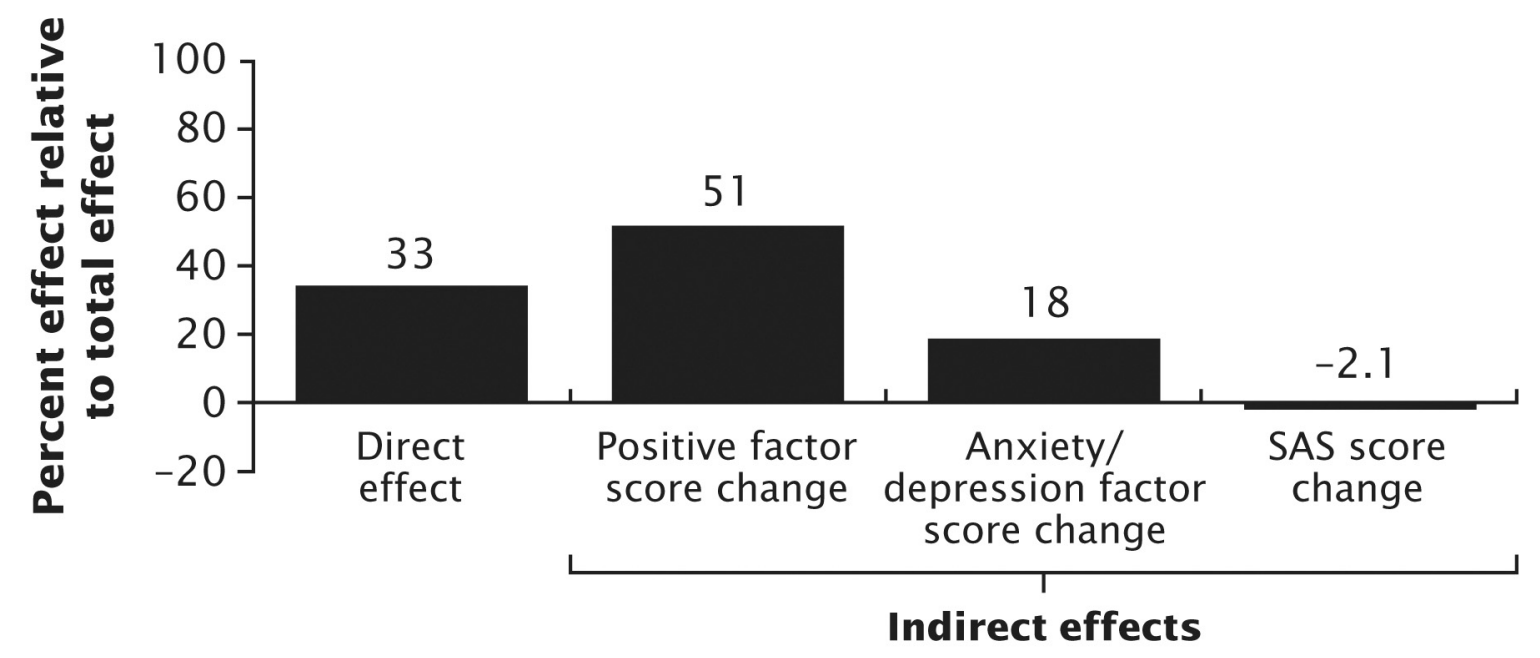

Figure 4 Percent of the total treatment effect of direct and indirect effects contributing to change in the negative factor score at end point, all paliperidone ER doses combined (Canuso et al 2006a). The Path analysis results suggest a 33\% direct effect of the combined paliperidone groups over placebo on the negative symptom factor score. Among those domains studied, the path analysis showed that the largest contributions to the change in negative symptoms were from the indirect effect mediated through the change in the positive factor score (51\%), followed by anxiety/depression (18\%), with little contribution made by changes in SAS scores ( $2.1 \%)$.

Abbreviations: ER, extended release; SAS, Simpson-Angus Rating Scale. 
scores, PSP scale scores and CGI-S scores (Canuso et al 2007; Dirks et al 2007). The additional improvements in symptoms and functioning observed following a switch to paliperidone ER may be attributed to the continued use of antipsychotic medication over time.

\section{Recurrence prevention}

Once an acute exacerbation has been controlled, the focus of treatment becomes prevention of recurrence, preservation of functioning and minimization of AEs associated with long-term medication exposure. To this end, Kramer et al conducted a randomized, double-blind, placebo-controlled, parallel-group, long-term study to assess the efficacy of paliperidone ER (flexibly dosed between 3-15 mg/day) compared with placebo (Kramer et al 2007a). The design involved 5 phases (Figure 5). The primary outcome measure was time to first recurrence in the double-blind phase. Recurrence was defined according to a series of pre-defined criteria (Table 2). Secondary outcome measures were change from baseline to end point on the PANSS total and CGI-S scores. Safety measures included self-reported AEs, SAS scores, Abnormal Involuntary Movement Scale (AIMS) scores, Barnes Akathisia Rating
Scale (BARS) scores, clinical laboratory evaluations, weight and electrocardiograms. The initial dose of paliperidone ER ( $9 \mathrm{mg}$ ) was selected based on pre-clinical data and was made prior to the analysis of any pivotal study data.

The modal daily dose of paliperidone ER received during the double-blind phase was $9 \mathrm{mg}$ by 39 (38\%) patients, $15 \mathrm{mg}$ by 29 (28\%) patients, $12 \mathrm{mg}$ by 23 (22\%) patients, $6 \mathrm{mg}$ by 9 (9\%) patients, and $3 \mathrm{mg}$ by 4 (4\%) patients (Kramer et al 2006). Based on the pre-defined criteria (Table 2), recurrence occurred in $52 \%$ of ITT patients on placebo and $22 \%$ on paliperidone ER. Moreover, $25 \%$ of patients experienced a recurrence on placebo at 23 days versus 83 days for those on paliperidone ER. Discontinuations in the double-blind phase were higher, however, in the paliperidone ER group $(n=20 ; 19 \%)$ compared with placebo $(n=8 ; 8 \%)$. Subject choice contributed to this difference (12 on paliperidone ER versus 0 on placebo) (Kramer et al 2007a).

The PANSS total scores during the run-in and stabilization phases went from $93.4 \pm 11.2$ to $52.2 \pm 11.0$ for the ITT population (Kramer et al 2006). During the run-in and stabilization phases, AEs were rated as mild or moderate in severity (Kramer et al 2006). The most frequent AEs

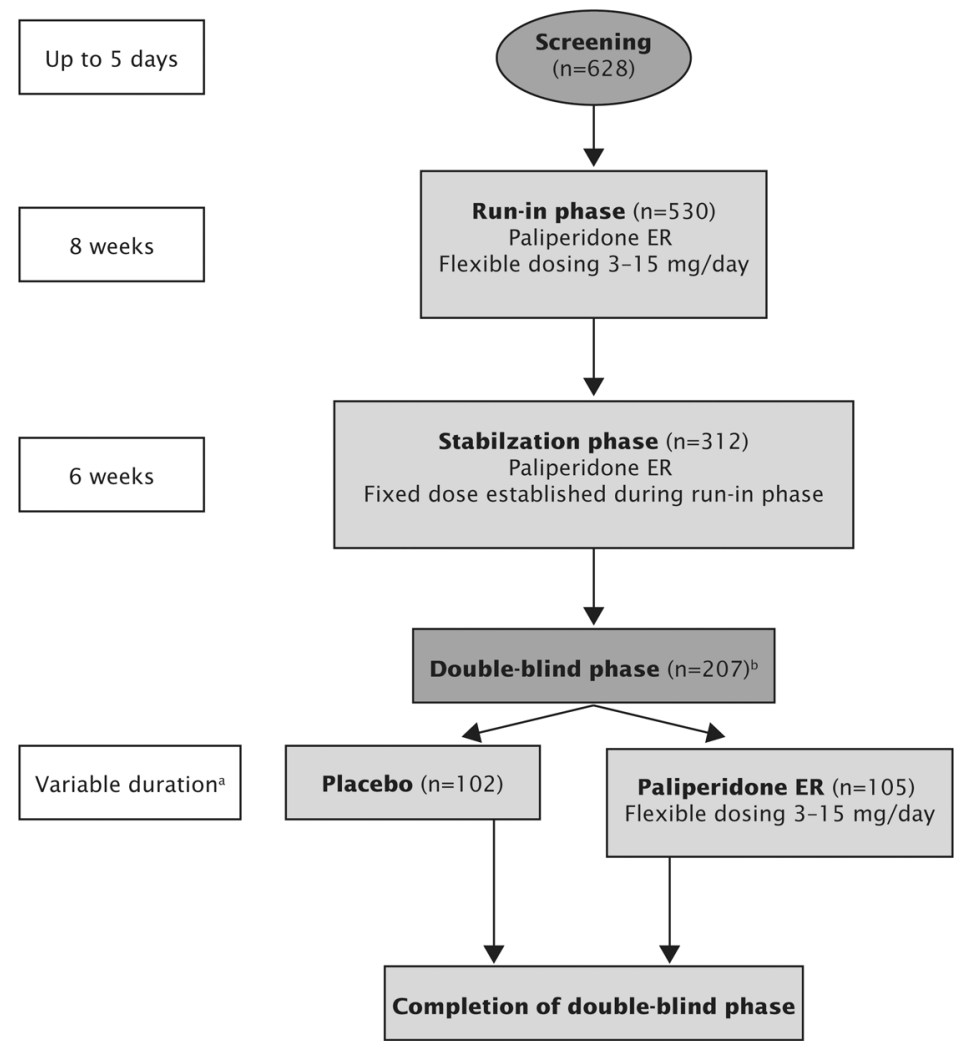

Figure 5 Study design to assess efficacy of paliperidone ER compared with placebo in relation to recurrence (Kramer et al 2007a). ${ }^{U} \mathrm{P}$ to 24 weeks after last subject randomized, or 86 recurrence events, with preplanned interim analysis at 43 events; 'bntent-to-treat population $(n=205)$, I patient lost to follow up, another patient did not receive any double-blind medication; 'Patients who were in the study at the time of its termination were considered to have completed the entire course of the study. 
Table 2 Definitions of recurrence (Kramer et al 2007a)

$\begin{array}{ll}\begin{array}{l}\text { Hospitalization } \\ \text { PANSS score }\end{array} & \begin{array}{l}25 \% \text { increase for two consecutive } \\ \text { days if baseline score was }>40 \\ 10 \text { point increase, if baseline score was } \leq 4\end{array} \\ \begin{array}{l}\text { Deliberate self-injury } \\ \text { and/or violent behavior }\end{array} & \\ \begin{array}{l}\text { Suicidal or homicidal } \\ \text { ideation }\end{array} & \\ \text { CGI-S score } & \begin{array}{l}\text { Score } \geq 4 \text { for } 2 \text { consecutive days } \\ \text { if baseline score was } \leq 3\end{array} \\ & \begin{array}{l}\text { Score } \geq 5 \text { for } 2 \text { consecutive days } \\ \text { if baseline score was } 4 \\ \geq 5 \text { on any item for } 2 \text { consecutive days }\end{array} \\ \text { PANSS items (PI, P2, P3, } & \begin{array}{l}\geq 6 \text { on any item for } 2 \text { consecutive days } \\ \text { P6, P7, G8) score }\end{array} \\ & \text { if baseline score was } 4\end{array}$

Abbreviations: CGI-S, Clinical Global Impression-Severity; PANSS, Positive and Negative Syndrome Scale.

(occurring in $\geq 10 \%$ of patients) included tremor (16\%), headache (14\%), hyperkinesia (12\%) and insomnia (10\%). Thirty SAEs occurred and were usually related to the psychotic disorder. They included psychosis (19 patients; 4\%), suicide attempt (4 patients; $1 \%$ ), agitation ( 2 patients; $1 \%$ ) and aggressive reaction (4 patients; <1\%) (Kramer et al 2006).

At the end of the double-blind phase, CGI-S scores of "mild/ very mild/not ill" were observed in $73 \%$ of the paliperidone ER group and $46 \%$ of the placebo group (Kramer et al 2006).

The authors concluded that paliperidone ER (flexibly dosed at $3-15 \mathrm{mg} /$ day) was superior to placebo in preventing recurrence after stabilization. In general, it was also well tolerated with longer-term exposure.

\section{Safety and tolerability of paliperidone ER}

Meltzer et al summarized the safety and tolerability for the three acute efficacy trials with paliperidone ER (Meltzer et al 2006). Overall, TEAEs were reported in 66\%$77 \%$ of patients in the paliperidone groups compared with $66 \%$ of patients in the placebo group. The most commonly observed AEs occurring in $\geq 5 \%$ of patients in any group included headache, anxiety and insomnia (See Table 3 ). SAEs were comparable in the paliperidone ER (5\%-6\%) and placebo groups $(6 \%)$.

\section{Extrapyramidal symptoms}

In the Meltzer pooled analysis (Meltzer et al 2006) the incidence of EPS was comparable between the paliperidone ER $3 \mathrm{mg}$ and $6 \mathrm{mg}$ groups (13\% and $10 \%$, respectively) and placebo (11\%). In the paliperidone ER (9 mg and $12 \mathrm{mg}$ ) groups, the incidence of EPS-related AEs was approximately doubled ( $25 \%$ and $26 \%$ of patients, respectively). Based on outcomes of the SAS, AIMS and BARS scales, the majority of paliperidone ER-treated patients did not experience any changes in movement disorders. It should be noted that the pivotal studies were fixed-dose trials; patients randomized to 9 or $12 \mathrm{mg}$ /day were not titrated upward from $6 \mathrm{mg} /$ day.

\section{Metabolic and endocrine effects}

Paliperidone ER (3-15 mg) produced a mean bodyweight change of $\leq 2 \mathrm{~kg}$ over the 6-week study period. Moreover, there were no clinically meaningful changes in glucose, insulin and blood lipid levels (Meyer et al 2006).

Kramer et al presented pooled data $(n=1083)$ from three open-label, 52-week paliperidone extension studies dosed between $3-15 \mathrm{mg} /$ day (starting dose $=9 \mathrm{mg}$ ). The mean duration of study participation was 232 days and the mean modal dose of paliperidone was $10.1 \mathrm{mg} /$ day. The serum glucose mean change to end point was $0.20 \pm 01.27 \mathrm{mmol} / \mathrm{L}$. Four patients had glucose elevations above the normal value and one had a glucose level below the normal range. The mean end point change in insulin levels was $2.8 \pm 36.99$ $\mu / \mathrm{mL}$. These glucose and insulin changes were similar to those observed during the double-blind studies. There were no increases observed in the mean change in triglycerides, total cholesterol, low-density cholesterol or high-density cholesterol observed. The mean end point changes in body weight and body mass index (BMI) were $1.1 \pm 5.47 \mathrm{~kg}$ and $0.4 \pm 1.96 \mathrm{~kg} / \mathrm{m}^{2}$, respectively. Those patients with a lower initial BMI $\left(<18.5 \mathrm{~kg} / \mathrm{m}^{2}\right.$ at baseline) were more likely to gain weight compared with those with a higher initial BMI ( $>25 \mathrm{~kg} / \mathrm{m}^{2}$ at baseline). The overall incidence of glucoserelated AEs was $<1 \%$, while the percentage of patients experiencing a weight increase-related $\mathrm{AE}$ was $5 \%$. No patients discontinued due to glucose-related AEs or weight gain (Kramer et al 2007b).

Paliperidone ER elevates prolactin levels in a fashion similar to risperidone. In this context, some studies have found a statistically significant correlation between serum 9-OH risperidone and prolactin level elevation (Melkersson 2006; Knegtering 2005). Conversely, others have not demonstrated a correlation between serum prolactin levels and plasma concentrations of risperidone, 9-OH risperidone, or the active moiety, following oral administration of risperidone (Zhou 2006). In the acute trials, potential prolactinrelated AEs were reported in 1\%-2\% of subjects receiving placebo or paliperidone ER (3-12 mg/day), and $4 \%$ in those receiving paliperidone ER ( $15 \mathrm{mg} /$ day $)$. 
Table 3 Incidence of treatment-emergent adverse events occurring in $\geq 5 \%$ of patients in any treatment group in the safety analysis set (Meltzer et al 2006)

\begin{tabular}{|c|c|c|c|c|c|c|}
\hline & $\begin{array}{l}\text { Placebo } \\
- \\
(n=355) n(\%)\end{array}$ & $\begin{array}{l}\text { Paliperidone } \\
\text { ER } 3 \mathrm{mg} \\
(\mathrm{n}=127) \mathrm{n}(\%)\end{array}$ & $\begin{array}{l}\text { Paliperidone } \\
\text { ER } 6 \text { mg } \\
(\mathrm{n}=235) \mathrm{n}(\%)\end{array}$ & $\begin{array}{l}\text { Paliperidone } \\
\text { ER } 9 \mathrm{mg} \\
(\mathrm{n}=246) \mathrm{n}(\%)\end{array}$ & $\begin{array}{l}\text { Paliperidone } \\
\text { ER I } 2 \mathrm{mg} \\
(\mathrm{n}=242) \mathrm{n}(\%)\end{array}$ & $\begin{array}{l}\text { Paliperidone } \\
\text { ER I5 mg } \\
(\mathrm{n}=\mid 13) \mathrm{n}(\%)\end{array}$ \\
\hline TEAEs in total & $235(66)$ & $91(72)$ & $156(66)$ & $|7|(70)$ & $184(76)$ & $87(77)$ \\
\hline \multicolumn{7}{|c|}{ Nervous system disorders } \\
\hline Headache & $42(12)$ & $14(11)$ & $29(12)$ & $34(14)$ & $35(14)$ & $20(18)$ \\
\hline Akathisia & $14(4)$ & $5(4)$ & $7(3)$ & $20(8)$ & $23(10)$ & $11(10)$ \\
\hline \multicolumn{7}{|l|}{ disorder } \\
\hline Somnolence & $12(3)$ & $6(5)$ & $8(3)$ & $17(7)$ & II (5) & $7(6)$ \\
\hline Dizziness & $14(4)$ & $7(6)$ & II (5) & II (4) & $12(5)$ & $7(6)$ \\
\hline Sedation & $13(4)$ & $\mathrm{I}(\mathrm{I})$ & $12(5)$ & $8(3)$ & $15(6)$ & $2(2)$ \\
\hline \multicolumn{7}{|l|}{ Psychiatric disorders } \\
\hline Insomnia & $5 I(4)$ & $14(11)$ & $29(12)$ & $35(14)$ & $26(11)$ & $14(12)$ \\
\hline Anxiety & $29(8)$ & $12(9)$ & $16(7)$ & $14(6)$ & II (5) & $9(8)$ \\
\hline Agitation & $28(8)$ & $7(6)$ & $17(7)$ & $13(5)$ & $13(5)$ & $3(3)$ \\
\hline Psychotic disorders & $16(5)$ & $5(4)$ & $6(3)$ & $7(3)$ & $4(2)$ & $4(4)$ \\
\hline \multicolumn{7}{|c|}{ Gastrointestinal disorders } \\
\hline Nausea & $19(5)$ & $8(6)$ & $9(4)$ & $10(4)$ & $10(4)$ & $2(2)$ \\
\hline Vomiting & $17(5)$ & $2(2)$ & $6(3)$ & $9(4)$ & $12(5)$ & $8(7)$ \\
\hline Constipation & $20(6)$ & $7(6)$ & $8(3)$ & $7(3)$ & $7(3)$ & $4(4)$ \\
\hline Dyspepsia & $14(4)$ & $3(2)$ & $6(3)$ & $5(2)$ & $12(5)$ & $6(5)$ \\
\hline \multicolumn{7}{|l|}{ Cardiac disorders } \\
\hline Tachycardia & $10(3)$ & $3(2)$ & $17(7)$ & $18(7)$ & $18(7)$ & $2(2)$ \\
\hline Sinus tachycardia & $15(4)$ & II (9) & $9(4)$ & $10(4)$ & $17(7)$ & $8(7)$ \\
\hline \multicolumn{7}{|l|}{ Investigations } \\
\hline $\begin{array}{l}\text { Electrocardiogram QT } \\
\text { corrected interval prolo }\end{array}$ & $9(3)$ & $4(3)$ & $9(4)$ & $7(3)$ & $12(5)$ & $4(4)$ \\
\hline
\end{tabular}

Abbreviations: ER, extended release;TEAE, treatment-emergent adverse event.

In the Marder et al study, potential prolactin-related AEs were experienced by 4 patients $(0.9 \%)$, including 3 men ( 2 patients on placebo, 1 patient on paliperidone ER $12 \mathrm{mg}$ ), and 1 woman (on paliperidone ER $12 \mathrm{mg}$ ), but did not result in study discontinuation. Potential prolactin-related AEs were impotence and abnormal sexual function with placebo; and nonpuerperal lactation and decreased libido with paliperidone ER $12 \mathrm{mg}$. Elevations from baseline prolactin levels were observed at Day 15 , and subsequently decreased but not to within normal range by the end point (Table 4) (Marder et al 2007).

In the Kane et al study, potential prolactin-related AEs (including galactorrhea, gynecomastia, amenorrhea, anorgasmia and abnormal sexual function) were reported by $1 \%$ of patients -3 men ( 1 in each of the paliperidone ER groups) and 4 women ( 1 in each of the paliperidone ER groups and 1 in the olanzapine group) - and resulted in 2 study discontinuations. Mean plasma prolactin levels increased from $17.4 \pm$ $19.9 \mathrm{ng} / \mathrm{mL}$ at baseline to $45.3 \pm 23.19 \mathrm{ng} / \mathrm{mL}$ at end point in the male patients on paliperidone ER, and from $38.03 \pm$ $57.0 \mathrm{ng} / \mathrm{mL}$ at baseline to $124.5 \pm 65.5 \mathrm{ng} / \mathrm{mL}$ at end point in the female patients on paliperidone ER. In contrast, mean prolactin levels decreased from baseline to end point in male and female subjects on placebo or olanzapine.

In the Davidson et al study, prolactin-related AEs (nonpuerperal lactation) and potential prolactin-related AEs (anorgasmia, impotence and decreased libido) were reported in 3 men ( 2 in the paliperidone ER $15 \mathrm{mg}$ group and 1 in the olanzapine group) and 3 women ( 2 in the paliperidone ER $15 \mathrm{mg}$ group and 1 in the paliperidone ER $3 \mathrm{mg}$ group). None of these events were considered severe or led to study discontinuation. Plasma prolactin levels increased from 16.5 $\pm 17.5 \mathrm{ng} / \mathrm{mL}$ at baseline to $42.9 \pm 29.1 \mathrm{ng} / \mathrm{mL}$ at end point in the males on paliperidone ER and from $36.6 \pm 68.8 \mathrm{ng} / \mathrm{mL}$ at baseline to $120.2 \pm 89.6 \mathrm{ng} / \mathrm{mL}$ at end point in the females on paliperidone ER (Davidson et al 2007). Again, mean prolactin levels decreased from baseline to end point in male and female patients on placebo or olanzapine (Davidson et al 2007).

When considering the effects of paliperidone ER on serum prolactin levels and the occurrence of potentially prolactin-related AEs, it must be recognized that these were spontaneously reported during the clinical studies, rather than as part of a systematic assessment. This may suggest 
Table 4 Changes in prolactin levels (baseline to endpoint) (Marder et al 2007)

\begin{tabular}{|c|c|c|c|c|}
\hline \multirow[t]{2}{*}{ Treatment group } & \multicolumn{2}{|c|}{$\begin{array}{l}\text { Mean } \pm \text { SD prolactin levels }(\mathrm{ng} / \mathrm{mL}) \\
\text { (males) }\end{array}$} & \multicolumn{2}{|c|}{$\begin{array}{l}\text { Mean } \pm \text { SD prolactin levels }(\mathrm{ng} / \mathrm{mL}) \\
\text { (females) }\end{array}$} \\
\hline & Baseline & Endpoint & Baseline & Endpoint \\
\hline Placebo & $12.0 \pm 10.0$ & $12.4 \pm 11.8$ & $20.3 \pm 24.3$ & $25.3 \pm 31.8$ \\
\hline Paliperidone ER (6 mg) & $13.4 \pm 1 \mid .4$ & $36.2 \pm 33.6$ & $23.4 \pm 21.4$ & $104.8 \pm 77.5$ \\
\hline Paliperidone ER (12 mg) & $15.6 \pm 19.6$ & $39.9 \pm 26.9$ & $35.4 \pm 43.4$ & $111.8 \pm 74.9$ \\
\hline Olanzapine & $14.4 \pm 18.8$ & $18.4 \pm 16.5$ & $17.0 \pm 10.9$ & $22.9 \pm 14.4$ \\
\hline
\end{tabular}

Abbreviations: Safety analysis set; SD, standard deviation.

that the frequency of prolactin-related AEs associated with paliperidone ER therapy is underestimated.

\section{Cardiac effects}

Paliperidone causes a modest increase in the corrected QT interval (QTc). During the three acute trials, 15 QT measurements were taken, including one measurement at the time of expected peak paliperidone ER serum concentration. The incidence of QTc prolongation on paliperidone ER ranged from $3 \%$ to $5 \%$ compared with $3 \%$ on placebo. Furthermore, there were no subjects who experienced a QTc interval $\geq 500$ msec. More specifically, in the Marder et al study, $1(1 \%)$ patient on placebo, $2(2 \%)$ patients on the $6 \mathrm{mg}$ dose and 1 (1\%) patient on the $12 \mathrm{mg}$ dose had a prolonged maximum post-baseline QTcLD (QTc linear derived) assessment (ie, $>450 \mathrm{~ms}$ for males and $>470 \mathrm{~ms}$ for females). In the Kane et al study no patient on paliperidone ER had a prolonged maximum post-baseline QTcLD assessment. In the Davidson et al study no patients on paliperidone ER had a post-baseline QTcLD interval exceeding $500 \mathrm{~ms}$.

Two additional studies were conducted to assess the cardiac effects of paliperidone ER. The first was a controlled study with moxifloxacin (400 $\mathrm{mg}$ dose) as an active control and a supratherapeutic dose of paliperidone IR, which provides at least twice the maximum exposure of paliperidone ER $12 \mathrm{mg}$. The $4 \mathrm{mg}$ and $8 \mathrm{mg}$ doses of paliperidone IR were associated with a QTc increase of $6.8 \mathrm{msec}$ and $12.3 \mathrm{msec}$, respectively (INVEGA package insert 2007). The second study was a randomized, double-blind, placebo-controlled study evaluating the QTc intervals of patients treated with paliperidone and quetiapine (Hough et al 2007). In this study, paliperidone ER (12 or $18 \mathrm{mg} /$ day) and quetiapine ( $400 \mathrm{mg}$ twice daily) resulted in minimal QTc prolongation. Mean changes in QTcLD were paliperidone ER $12 \mathrm{mg} /$ day, $1.71 \mathrm{~ms}$ versus quetiapine $800 \mathrm{mg}$ /day, $6.06 \mathrm{~ms}$; paliperidone ER $18 \mathrm{mg} /$ day, $3.70 \mathrm{~ms}$; and quetiapine $800 \mathrm{mg} / \mathrm{day}, 5.57 \mathrm{~ms}$.

Paliperidone ER should be avoided in combination with other drugs that are known to prolong the QTc duration including Class 1A (eg, quinidine, procainamide) or Class III (eg, amiodarone, sotalol) anti-arrhythmic medications; antipsychotic medications (eg, chlorpromazine, thioridazine); antibiotics (eg, gatifloxacin, moxifloxacin); or any other class of medications known to prolong the QTc interval. Additionally, paliperidone ER should also be avoided in patients with congenital long QT syndrome or a history of cardiac arrhythmias (INVEGA package insert 2007).

\section{Long-term safety of paliperidone ER}

During the double-blind phase of the recurrence study, TEAEs occurred at a similar rate in both treatment arms $(35 \%$ in the paliperidone ER group and $40 \%$ in the placebo group) (Kramer et al 2007a). Only three of these (psychosis, aggressive reaction, and insomnia) occurred in $\geq 5 \%$ of subjects. Psychosis and aggressive reaction were more frequent in the placebo compared with the paliperidone ER group (23\% versus 7\% and $6 \%$ versus $1 \%$, respectively). Extrapyramidal symptoms were reported in $3 \%$ of patients in the placebo group and $7 \%$ of patients in the paliperidone ER group. There were no occurrences of glucose-related AEs. Also, weight gain was modestly higher in the paliperidone ER versus placebo group (ie, $1.8 \pm 4.8 \mathrm{~kg}$ versus $0.2 \pm 5.0 \mathrm{~kg}$, respectively) (Kramer et al 2006). Prolactin-related AEs occurred in $4 \%$ of patients in the paliperidone ER group and $\%$ of the placebo group. In terms of tachycardia, there were no differences between the two groups ( $7 \%$ in the paliperidone ER group and $2 \%$ in the placebo group). Four subjects (2\%) discontinued due to AEs ( 1 on placebo; 3 on paliperidone ER) (Kramer et al 2006). Additionally, a favorable long-term safety profile was demonstrated in a pre-specified, pooled analysis of the three 52-week, open-label extension phases from the Kane et al, Davidson et al and Marder et al studies (Eerdekens et al 2007). No notable changes in EPS symptoms were observed in terms of SAS, AIMS or BARS median scores. Potential prolactin-related AEs were reported in $\leq 1 \%$ (except for amenorrhea [4\%]) of subjects who received paliperidone ER during both the double-blind and open-label phases of the studies. Mean plasma prolactin levels were also 


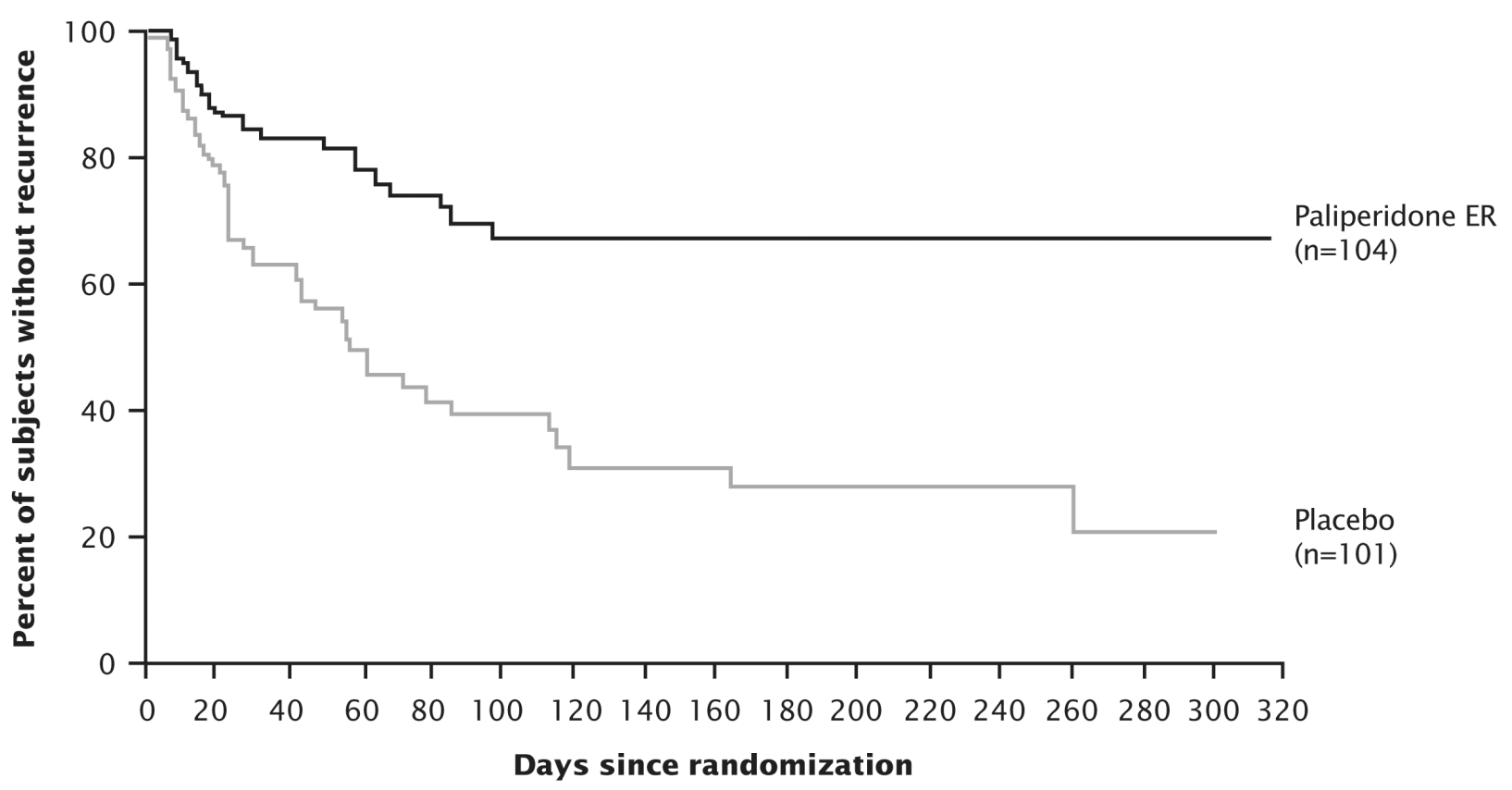

Figure 6 Kaplan-Meier plot of time to recurrence - final intent-to-treat analysis set (Kramer et al 2007a). Kaplan-Meier estimates for the time to recurrence of schizophrenia symptoms in the double-blind (final analysis) group for paliperidone ER versus placebo.

Abbreviations: ER, extended release.

Reprinted with permission from Kramer M, Kushner S,Vijapurkar U, et al 2007. Paliperidone extended-release tablets for prevention of symptom recurrence in patients with schizophrenia:A randomized, double-blind, placebo-controlled study.J Clin Psychopharmacol, 27:6-14. Copyright () 2007 Lippincott, Williams and Wilkins.

observed to decrease over the course of the open-label phases. Only $1 \%$ of subjects had a prolonged maximum post-baseline QTcLD $\geq 450 \mathrm{~ms}$, and one patient experienced a maximum post-baseline QTcLD $\geq 480 \mathrm{~ms}$.

\section{Future directions}

Additional efficacy studies in acute mania and for the longterm treatment of schizophrenia are in progress. Furthermore, a placebo-controlled, head-to-head comparison of paliperidone ER and quetiapine for treatment of an acute exacerbation of schizophrenia is ongoing. In the absence of a risperidone/paliperidone head-to-head trial, a "virtual" analysis comparing 6-week data from double-blind, placebo-controlled studies of risperidone and paliperidone $\mathrm{ER}$ in patients suffering an acute exacerbation of schizophrenia has been conducted (Schooler et al 2006). This post hoc analysis attempted to assess how paliperidone ER compares to risperidone with respect to efficacy and tolerability. Patient populations were matched based on age (18-65 years); exposure to a conventional antipsychotic within 90 days of study entry; and treatment with paliperidone ER (3-12 mg/day), risperidone (2-6 mg/day) or placebo. A total of 1103 subjects were included in the analysis. The primary outcome measure was the change in PANSS total score over 6 weeks. Tolerability comparators were weight gain and reported AEs (adjusted for cross-program differences identified in placebo groups). Baseline characteristics of the two placebo groups were similar, with the exception of a larger proportion of males included in the risperidone program and more Caucasians in the paliperidone ER program. The most frequently utilized schizophrenia treatment doses were defined as 4-6 mg/day of risperidone and 6-12 $\mathrm{mg} /$ day of paliperidone ER and are the most relevant for comparison. As such, there were 174 patients in the risperidone group and 275 patients in the paliperidone ER dose groups. Within these two groups, study completion rates $(65.5 \%$ for risperidone and $67.7 \%$ for paliperidone ER) and mean PANSS changes (19.7 for risperidone and 19.0 for paliperidone ER) were similar. Adverse event rates demonstrated a lower rate of akathisia, restlessness, anxiety, insomnia, somnolence, dizziness and gastrointestinal effects in the paliperidone ER group compared with the risperidone group. Weight gain was slightly higher in the risperidone group $(1.3 \pm 3.7 \mathrm{~kg})$ compared with paliperidone ER $(0.7 \pm 2.7 \mathrm{~kg})$.

\section{Conclusion}

Paliperidone ER is indicated for the treatment of schizophrenia. Based on the results of the acute clinical trials, the recommended starting and target dose is $6 \mathrm{mg}$ once daily in the 
morning. While higher doses may produce further benefit, this strategy is mitigated by a dose-related increase in some AEs, such as QTc prolongation, EPS and weight gain. The recommended dosing range is 3-12 mg per day. Dose adjustments of $3 \mathrm{mg}$ increments or decrements are recommended at intervals of not less than 5 days. For individuals with moderate-to-severe renal impairment (ie, creatinine clearance 10 to $<50 \mathrm{ml} / \mathrm{min}$ ) the maximum recommended dose is $3 \mathrm{mg}$ per day.

Paliperidone ER (9-OH risperidone), utilizing the OROS delivery technology, has demonstrated efficacy and a favorable tolerability profile in the treatment of schizophrenia. This delivery system can be adapted to the specific properties of a drug to provide more stable plasma concentrations. In addition, paliperidone ER's limited hepatic metabolism should lower the risks of related hepatic drug-drug or drug-disease pharmacokinetic interactions. While there are long-term data from the recurrence prevention study (Kramer et al 2007b), further data are needed to assess its potential role for long-term management (including a long-acting formulation) and for other disorders (eg, bipolar mania, schizoaffective disorder).

In the US, risperidone will be available as a generic product midsummer 2008. It is anticipated that the availability of this generic, second generation antipsychotic will impact the utilization of paliperidone ER, as well as other second generation antipsychotics. In particular, managed care may implement increased restrictions on other second generation antipsychotics in an attempt to increase utilization of generic risperidone. Unfortunately, this purely economic focus jeopardizes the provision of timely and appropriate clinical care to patients who may benefit more from an alternate agent. Thus, with the pending generic formulation of risperidone, cost differences may impact the use of paliperidone ER. While the recently presented "virtual" head-to-head analysis provides some useful comparison data to risperidone, adequately powered trials that compare paliperidone ER with other second generation antipsychotics (including risperidone) would be very useful in this context. Potential benefits over risperidone that may mitigate this issue, however, include the minimal hepatic metabolism in a patient population with substantial liver problems (eg, hepatitis C, alcohol-related) and frequent concurrent use of other hepatically metabolized psychotropic and non-psychotropic agents. In addition, paliperidone palmitate is currently in Phase III development as an aqueous, nanotechnology-based, long-acting injectable (LAI) formulation (Rosen and Abribat 2005). This formulation will be different than the currently available risperidone LAI. The first two paliperidone palmitate injections will be administered as a loading dose within 7 days of initiation. Thereafter, injections will be given monthly. This eliminates the need for the 3-week (or more) oral antipsychotic supplementation required with risperidone LAI. Furthermore, the ease of administration will be enhanced with the paliperidone LAI via utilization of pre-filled syringes that do not require refrigeration.

\section{Acknowledgments}

The authors would like to thank Samantha Taylor, PhD, Medicus International, for her editorial assistance with the manuscript. The editorial support for this manuscript was supported by funding from Ortho-McNeil Janssen Scientific Affairs, LLC.

\section{Disclosures}

Philip G Janicak is a member of the speakers bureau for Abbott, AstraZeneca, Bristol-Myers Squibb, Janssen and Pfizer, has received grant support from AstraZeneca, Bristol-Myers Squibb, Janssen, Neuronetics, Solvay and sanofi-aventis, and is an advisor/consultant to AstraZeneca, Bristol-Myers Squibb, Janssen, Neuronetics and Solvay. The paper was principally written while co-author Elizabeth A Winans was employed within Scientific Affairs, Ortho McNeil Janssen, LLC, Chicago, IL, USA.

\section{References}

Canuso C, Bossie C, Turkoz I, et al. 2006a. Direct and indirect effects of paliperidone extended-release tablets on negative symptoms of schizophrenia. Int J Neuropsychopharmacol, 9:S280.

Canuso C, Youssef E, Dirks B, et al. 2006b. Paliperidone extended-release in severely ill patients with schizophrenia. Presented at the Institute on Psychiatric Services, October 5-8 2006, New York, NY, USA.

Canuso C, Youssef E, Bossie C, et al. 2007. Effects of paliperidone ER in patients with schizophrenia previously treated with risperidone. Presented at the International Congress on Schizophrenia Research, 28 March-1 April 2007, Colorado Springs, CO, USA.

Carney C, Jones L, Woolson RF. 2006. Medical comorbidity in women and men with schizophrenia. J Gen Intern Med, 21:1133-7.

Conley R, Gupta SK, Sathyan G. 2006. Clinical spectrum of the osmoticcontrolled release oral delivery system $\left(\mathrm{OROS}^{\circledR}\right)$, an advanced oral delivery form. Curr Med Res Opin, 22:1879-92.

Davidson M, Emsley R, Kramer M et al. 2007. Efficacy, safety and effect on functioning of paliperidone extended-release tablets in the treatment of acute schizophrenia: an international 6-week placebo-controlled study. Schizophrenia Res, 93:117-30.

Dirks B, Eerdekens M, Turkoz I, et al. 2006. Efficacy of paliperidone extended-release tablets in patients with schizophrenia and predominant negative symptoms. Int $J$ Neuropsychopharmacol, 9:S162.

Dirks B, Youssef E, Bossie C, et al. 2007. Effects of paliperidone ER in patients with schizophrenia previously treated with olanzapine. Presented at the International Congress on Schizophrenia Research, March 28-April 1 2007, Colorado, USA.

Eerdekens M, Kramer M, Lane R, et al. 2007 Efficacy and tolerability of oral paliperidone extended-release tablets in the treatment of acute schizophrenia: Pooled data from three 52-week, open-label extension studies. Poster presented at the International Congress on Schizophrenia Research, March 28-April 1 2007, Colorado Springs, CO, USA. Poster number: 290 . 
Guy W. 1976. Clinical Global Impressions Scale. ECDEU Assessment manual. 218-22.

Hough D, Kramer M, Cleton A, et al. 2007. A randomized, double-blind, placebo-controlled, parallel-group study evaluating QT/QTC intervals following administration of paliperidone ER and quetiapine in patients with schizophrenia or schizoaffective disorder. Presented at Society of Biological Psychiatry, May 17-19 2007, San Diego, CA, USA. Poster number: 858.

INVEGA package insert. 2007. Prescribing information for paliperidone extended-release [online]. Accessed June 2007. URL: http://www.janssen.com/active/janus/en_US/assets/common/company/pi/invega.pdf; jsessionid $=3$ HLH4CUHTOA0UCQPCCFTCOYKB2IIQNSC.

Janicak P, Davis J, Preskorn S, et al. 2006. Principles and Practice of Psychopharmacotherapy. 4th ed. Philadelphia: Lippincott Williams and Wilkins. p 81-182.

Kane J, Canas F, Kramer M, et al. 2007. Treatment of schizophrenia with paliperidone extended-release tablets: A 6-week placebo-controlled trial. Schizophrenia Res, 90:147-61.

Knegtering R, Baselmans P, Castelein S, et al. 2005. Predominant role of the 9-hydroxy metabolite of risperidone in elevating blood prolactin levels. Am J Psychiatry, 162:1010-2.

Kostic D, Bossie C, Turkoz I. 2006a. Paliperidone extended-release tablets in patients recently diagnosed with schizophrenia. Presented at Collegium Internationale Neuro-Psychopharmacologicum, July 9-13 2006, Chicago, IL, USA.

Kostic D, Bossie C, Turkoz I, et al. 2006b. Paliperidone extended-release tablets in patients recently diagnosed with schizophrenia. Int J Neuropsychopharmacol, 9:S161.

Kramer M, Kushner S, Vijapurkar U, 2006. Delaying symptom recurrence in patients with schizophrenia with paliperidone extended-release tablet: an international, randomized, double-blind, placebo-controlled study. Presented at the Collegium Internationale Neuro-Psychopharmacologicum, July 9-13 2006, Chicago, IL, USA.

Kramer M, Kushner S, Vijapurkar U. 2007a. Paliperidone extended-release tablets for prevention of symptom recurrence in patients with schizophrenia: a randomized, double-blind, placebo-controlled study. J Clin Psychopharmacol, 27:6-14.

Kramer M, Eerdekens M, Lane R, et al. 2007b. Metabolic outcomes, in terms of weight, glucose and lipid profiles, in patients with schizophrenia treated with paliperidone extended-release tablets for 52 weeks. Presented at the American Psychiatric Association Annual Meeting, May 19-24 2007, San Diego, CA, USA. Poster number: NR498.

Luthringer R, Staner L, Noel N. 2007. A double-blind, placebo-controlled, randomized study evaluating the effect of paliperidone extended release tablets on sleep architecture in patients with schizophrenia. Int Clin Psychopharm, 22:299-308.

Marder SR, Kramer M, Ford L, et al. 2007. Efficacy and safety of paliperidone extended-release tablets: results of a 6-week, randomized, placebo-controlled study. Biol Psychiatry, June 27; [Epub ahead of print] doi:10.1016/j.biopsych.2007.01.017.

Marder SR, Davis JM, Chouinard G. 1997. The effects of risperidone on the five dimensions of schizophrenia derived by factor analysis: combined results of the North American trials. J Clin Psychiatry, 58:538-46.

Melkersson KI. 2006. Prolactin elevation of the antipsychotic risperidone is predominantly related to its 9-hydroxy metabolite. Hum Psychopharmacol, 21:529-32.
Meltzer H, Kramer M, Gassmann-Mayer C, et al. 2006. Efficacy and tolerability of oral paliperidone extended-release tablets in the treatment of acute schizophrenia: pooled data from three 6-week placebo controlled studies. Int J Neuropsychopharmacol, 9:S225.

Meyer J, Kramer M, Lane R, et al. 2006. Metabolic outcomes in patients with schizophrenia treated with oral paliperidone extended-release tablets: pooled analysis of three 6-week placebo-controlled studies. Int $J$ Neuropsychopharmacol, 9:S282.

Morosini PL, Magliano L, Brambilla L. 2000. Development, reliability and acceptability of a new version of the DSM-IV Social and Occupational Functioning Assessment Scale (SOFAS) to assess routine social functioning. Acta Psychiatr Scand, 101:323-9.

Patrick D, Adriaenssen I, Morosini P, et al. 2006. Reliability, validity and sensitivity to change of the personal and social performance scale in patients with acute schizophrenia. Int J Neuropsychopharmacol, 9: S287-88.

Rosen H, Abribat T. 2005. The rise and rise of drug delivery. Nat Rev Drug Discov, 4:381-5.

Rossenu S, Cleton A, Talluri K, et al. 2007. Evaluation of the pharmacokinetics of an extended-release formulation of paliperidone with an immediate-release formulation of risperidone. Clin Pharm Ther, 81(Suppl 1):S62.

Rossenu SAC, Rusch S, Janssens S, et al. 2006. Extended-release formulation of paliperidone shows dose proportional pharmacokinetics. Presented at the American Association of Pharmaceutical Sciences Annual Meeting and Exposition, October 29-November 2 2006, San Antonio, TX, USA. Poster number: T3123.

Schooler N, Gharabawi G, Bossie C, et al. 2006. A virtual comparison of paliperidone ER and oral risperidone in patients with schizophrenia. Presented at the 45th Annual Meeting of the American College of Neuropharmacology, December 3-7 2006, Hollywood, FL, USA.

Thyssen A, Cleton A, Osselae NV. 2007. Effects of renal impairment on the pharmacokinetic profile of paliperidone extended-release tablets. Clin Pharm Ther, 81(Suppl 1):S63.

Thyssen A, Cleton A, Talluri K, 2006a. Absence of pharmacokinetic interaction between trimethoprim and paliperidone extended-release tablets in healthy subjects. Biol Psychiatry, 59:181-64.

Thyssen A, Crauwels H, Cleton A, et al. 2006b. Effects of hepatic impairment on the pharmacokinetics of paliperidone immediate-release. Presented at the 46th New Clinical Drug Evaluation Unit Annual Meeting, June 12-15 2006, Boca Raton, FL, USA.

Tzimos A, Samokhvalov V, Kramer M, et al. 2007. Safety and tolerability of oral paliperidone extended-release tablets in elderly patients with schizophrenia: A double-blind, placebo-controlled study with long-term open-label extension. Am J Geriatric Psych, in press.

Vermeir M, Boom S, Naessens I, et al. 2005. Absorption, metabolism and excretion of a single oral dose of 14C-paliperidone $1 \mathrm{mg}$ in healthy subjects. Eur Neuropsychopharmacol, 30:S191-92.

Zhou Z, Li X, Peng H, et al. 2006. Predominant role of the 9-hydroxy metabolite of risperidone in Chinese female patients with schizophrenia. Acta Pharmacol Sin, 27:381-6. 
